\title{
Importancia del desarrollo de los individuos y su incidencia en el comportamiento organizacional ${ }^{*}$
}

\section{Importance of developing individuals and its incidence} in the organizational behavior

\author{
María Clemencia Ardila Franco** \\ Ana Elsa Vargas Espinosa**
}

Artículo de reflexión

\section{Resumen}

El propósito de este artículo es determinar la importancia presente y futura que tiene para la organización el desarrollo integral previo de los individuos que se incorporarán a ella. La reflexión fue producto de la revisión de diferentes teorías, teniendo en cuenta aspectos como formación y relación equilibrada de los componentes intelectuales, psicológicos, espirituales y corporales del individuo. Donde la madurez, la integridad y los valores definen un crecimiento continuo en convicciones, discernimiento, compromiso, confianza y seguridad, que integrado a través de su visión futura, en los diferentes ambientes, roles y comportamientos laborales, potencian

* Artículo desarrollado en el marco de las líneas de formación en Liderazgo y Organizaciones de la Maestría en Gestión de Organizaciones de la Universidad Militar Nueva Granada.

** Profesional de planta, Instituto Nacional de Vías, Subdirección Red Nacional de Carreteras; Bogotá, Colombia. Correo electrónico: cardila@ invias.gov.co; clemencia.ardila@gmail.com

*** Docente tiempo completo, Universidad Militar Nueva Granada, Facultad Estudios a Distancia. Bogotá, Colombia. Correo electrónico: ana. vargas@unimilitar.com 
una evolución innovativa permanente. En consecuencia la organización debe proyectar y proponer diferentes retos a los colaboradores dentro de sus estrategias, sin olvidar que el punto de partida y las competencias de entrada son la plataforma de la productividad y competitividad dirigidas al logro de los ideales individuales, conjuntamente con los objetivos estratégicos organizacionales.

Palabras clave: desarrollo integral, crecimiento, innovación, competencias.

\section{Abstract}

The purpose of this paper is to determine the present and future importance for the organization that previous integral development of individuals who will join it, has.

This reflection was the result of reviewing different theories, taking into account aspects such as training and

\section{Introducción}

La educación del individuo es fundamental, ya que ella enmarca en muchas formas el futuro emocional conductual del mismo; la representación mental-espacial de los pensamientos de la persona se refleja a lo largo de la vida en emociones, las cuales le facilitarán el camino al éxito. Recordemos que desde la crianza se pueden observar los pequeños y grandes pasos que da un niño en lo que se refiere a autonomía, emociones, valores, ética, compromiso, confianza, liderazgo, creatividad, las cuales pueden ser incentivadas en forma positiva por sus padres, profesores, amigos, compañeros y familiares que se encuentran alrededor de él. balanced relationship between intellectual, psychological, spiritual and physical components of the individual, where maturity, integrity and values define a continuous growth in convictions, insight, commitment, trust and security. This integrated to its future vision in different work roles and behaviors as well as work scenarios permanently boost innovative development.

Consequently, the organization must plan and propose different challenges to employees within its own strategies, keeping in mind that the starting point and initial competencies are the platform of productivity and competitiveness towards the achievement of individual ideals, together with the achievement of organizational strategic objectives.

Keywords: integral development, growth, innovation, competencies.

En efecto, el proceso de crecimiento y desarrollo del individuo se produce por la sumatoria de una serie de factores definidos por el entorno de la persona, a través de la vida. En este orden de ideas es necesario tener claridad respecto de algunos conceptos, ya que el presente artículo tiene como objetivo determinar el nivel de importancia del desarrollo de los individuos y su incidencia en la productividad y competitividad de la organización, concentrándonos en un artículo de carácter reflexivo para el campo laboral, basado en las experiencias individuales que contrastadas con las grupales proporcionan un resultado óptimo para la organización.

De acuerdo con lo propuesto, es necesario partir de las principales cualidades y características del ser humano 
que marcan de una u otra forma sus potencialidades y que al combinarlas con las potencialidades de otros individuos logran proyectar un horizonte mutuo con metas y objetivos que permiten el desarrollo y crecimiento de todos.

En cuanto al desarrollo del individuo hay que estar atentos a su educación, para que él logre desarrollar nuevas ideas para alcanzar sus metas, ideas que le permitan equilibrarse, utilizando sus conocimientos, comprensión, autocorrección, confianza, autoestima, reelaboración de situaciones y enriquecimiento de las mismas, como herramientas consiguiendo el control de sí mismo y la realización de sus objetivos.

Cuando el niño va creciendo, el ideal es que llegue a tener una personalidad y actuar maduro; desde el punto de vista emocional, intelectual, integrando impulsos, instintos, necesidades, sentimientos, acciones y pensamientos. Conociéndose a sí mismo, reconociendo cuando sus actuaciones son adecuadas, con una actitud de cambio, cuyos planes y objetivos sean alcanzables manteniendo una capacidad de decisión óptima, una responsabilidad acorde con las situaciones y una fuerza de voluntad que lo lleven a ser constante en sus acciones, con motivaciones que le aseguren el éxito.

En el presente documento se establece una relación entre la buena formación del individuo y la incidencia que este puede tener en una organización y viceversa. Este tipo de relación no solo beneficia al individuo, sino a la organización; de tal manera que la organización tendrá que contemplar el comportamiento organizacional, la calidad de vida laboral, así como también los entornos intelectuales, sociales, humanos y laborales que lleven a la organización a ejercer un liderazgo efectivo. En otras palabras, la organización forma parte del cambio integral del individuo dentro del campo laboral, cuando desarrolla, propone e implementa normas, estímulos, incentivos, valores y recursos produciendo cambios sucesivos, que inciden en un cambio en la cultura organizacional día tras día. Llevando al individuo y a la organización al logro de los objetivos y a la obtención de las metas individuales y organizacionales.

A su vez, el individuo para satisfacer sus necesidades activa una serie de conductas que combinadas con las de los grupos a los que tiene acceso dentro de un ambiente complejo, proporcionan experiencias que harán que el individuo crezca y se proponga nuevas y mejores metas dentro de una construcción social continua.

Gary Becker (1975) afirma “...el mayor tesoro que tienen las empresas es el capital humano..." significa entonces, que el capital humano conformado por todas las habilidades del individuo, sumadas a la parte cognitiva, la espiritual, la de liderazgo, la de salud, la de calidad de sus hábitos, incidirán en la productividad de la organización positivamente; lo anterior, basado principalmente en la formación del conocimiento del individuo, en su expresión y actuación a través de acciones que transformarán a cada paso la cultura y comportamiento individual y organizacional, proporcionando un valor agregado a la sociedad.

Por lo tanto, concluye el artículo en que las organizaciones deberán propiciar la combinación de la integralidad del ser humano, con escenarios propicios tendientes al buen manejo y adaptación de los individuos, habilitándolo en la capacidad para responder ante una nueva situación en forma efectiva, dando como resultado una ventaja competitiva para la organización, estableciendo en forma regulada una dinámica evolutiva para el desempeño de la misma. 


\section{Referente teórico}

\subsection{Aspectos de análisis factor humano}

El proceso de crecimiento y desarrollo del individuo está proporcionado por el acopio de factores enmarcados por la educación recibida y las experiencias percibidas en los diferentes entornos a través del tiempo; por ello, es necesario revisar algunos aspectos de la condición humana que son importantes e inciden en el individuo y por ende en la organización en el momento de tomar decisiones y de establecer los caminos a seguir y las metas u objetivos por cumplir; teniendo en cuenta que estos en consecuencia llevarán a satisfacer las necesidades individuales en primera instancia y las corporativas en segunda.

\subsubsection{La autonomía}

Olivia Parra (2001, pp. 181-196) precisan que la autonomía en la niñez significa poder llegar a ser capaz de pensar por sí mismo, dado por un argumento inicial que es el respeto mutuo dentro de un contexto familiar y escolar. La autonomía, entonces, estará referida al actuar de acuerdo con sus criterios, conciencia y valores que llevarán al individuo a ser libre, se relaciona con su parte emocional y conductual; cuya base principal es la cercanía emocional en las relaciones entre padres e hijos, que permiten al individuo tener determinado grado de adaptabilidad o calidad de comunicación.

De la misma manera, existen otros razonamientos sobre la autonomía en el sentido de estar estrechamente enlazada con el poder, la asertividad, la autoestima que son reflejadas en la conducta humana y por ende en el desarrollo de los objetivos del individuo y de todas las situaciones que lo involucran, en especial si pertenece a una organización.
La autoestima, a su vez, indica si el individuo está o no complacido con él mismo; es donde crece la semilla de la autonomía, al permitirle enfrentar los riesgos y los trabajos desafiantes con visión de éxito. La autoestima incluye respeto por sí mismo, por los demás; reconocimiento por las labores ejecutadas, atención del individuo y de los que lo rodean, dando una base importante a su seguridad en la autonomía y el anticipo de los logros que obtendrá; implica crecimiento del individuo, conocimiento, desarrollo de su potencial, logro de sus metas y satisfacción de sus necesidades, como lo destacan Olivia, Parra (2001).

El ambiente de la organización por tanto le facilita al individuo un desarrollo de su autonomía dentro de un contexto muy complejo que conlleva una estructura organizacional, poder, comunicación, trabajo en equipo, procesos de cambio, clima organizacional, liderazgo y otros, ya que también dependerá en gran medida de la armonía con sus compañeros, de la motivación y de las características de desarrollo de la misma organización interrelacionada con el entorno.

\subsubsection{El desarrollo cognitivo}

Desde antes de nacer el ser humano vive diferentes etapas de desarrollo, afronta y supera situaciones a través de investigación e incrementa su capacidad mental adquiriendo nuevos conocimientos (construcción de procesos de pensamiento enlazados con resolución de problemas), alternando modelos mentales, promoviendo la comunicación (relación con los demás), aplicando valores desde y hacia el individuo (entorno), buscando su plenitud y creciendo como ser humano.

Piaget (1992) citado por Kohlberg, propone teorías del desarrollo cognitivo, que incluyen estados de desarrollo desde la infancia pasando por la adolescencia y llegando al adulto; experimentando reflejos innatos, incorporando 
nuevos conocimientos, organizando diseños conductuales, que con el tiempo se interiorizan y se convierten en pensamientos que transforman la personalidad. Sin olvidar que Piaget en su estudio integra además la parte orgánica, biológica y genética del individuo.

Una de las herramientas del desarrollo cognitivo es el lenguaje, Ausubel (1991) citado por Meyer y Allen, habla del lenguaje y sus soportes en la significatividad lógica y significatividad psicológica, desarrolladas una desde el interior del sujeto y la otra desde la estructura cognitiva del mismo, retenidas a través de la organización de esquemas jerarquizados; condiciones que llevan al individuo a propiciar el equilibrio tanto interno como externo.

La educación juega un papel muy importante en el desarrollo del individuo, al producir nuevos conocimientos; experiencia vivida desde cuando los padres enseñan a sus hijos las diferencias existentes entre los elementos de la realidad, activando las capacidades intelectuales, los pensamientos, sentimientos y emociones, generando pensamiento sistémico; a la vez que el individuo coordina y armoniza todos estos elementos.

Por su parte, la sociedad enseña a los individuos, a través del lenguaje verbal, sensorial y tecnológico, los conocimientos, el intelecto y la cultura cuando el individuo tiene contacto con otros individuos, trasmite nuevos conocimientos a otros contextos, generando un proceso unitario y uno global, mientras desarrolla su inteligencia, señalado por Vidal (2007).

Otra de las manifestaciones del desarrollo cognitivo es que la inteligencia ${ }^{1}$ se encuentra en cada individuo y este la desarrolla en forma parcial o plena en el transcurrir de la vida, de tal manera que la misma actúa en el individuo como mecanismo que lo coadyuva a establecer su equilibrio, el entorno social y el medio ambiente que lo rodea.

Gardner (2001) en su teoría de las inteligencias múltiples distingue varios tipos de inteligencia como son: la lingǘstica (capacidad de uso de las palabras de manera adecuada); inteligencia lógica (utiliza el hemisferio lógico resolviendo problemas); inteligencia musical (desarrolla el oído y las capacidades musicales); inteligencia espacial (usar e interrelacionar los colores, líneas, formas, figuras y espacios en tres dimensiones); inteligencia corporal y cenestésica (se emplean las expresiones controlando y coordinando los movimientos del cuerpo).

Además, existe también la inteligencia intrapersonal, referida a entenderse como ser humano, descubrirse y descubrir la capacidad creativa vinculada con las emociones; otra inteligencia es la interpersonal al percibir a los demás con empatía, identificar sus verdaderas capacidades y las de la gente del entorno; distinguiendo qué quiere ser como individuo, qué quiere la gente del entorno y qué está dispuesto a hacer por ellos. Emergiendo otros tipos de inteligencia como la referida al medio natural (desarrolla capacidades de organización y clasificación); hasta la situación del individuo respecto al cosmos, de acuerdo con lo dicho por Gardner (2001).

Dado lo anterior, la inteligencia estará entonces compuesta por la integralidad y combinación armónica equilibrada de todos los tipos de inteligencia mencionados anteriormente, naciendo un individuo estable, influenciado por registros mentales de su herencia educativa, cultura generacional, entorno social dentro del cual aprovechará

1 Myers, 2006 define la inteligencia como “... La capacidad para mostrar un comportamiento adaptativo orientado hacia objetivos, implica las cualidades necesarias para aprovechar la experiencia,

resolver problemas, razonar y afrontar con éxito desafios y alcanzar objetivos..." 
una inteligencia más que otra, sesgándose a la que le produce el comportamiento más adecuado.

$\mathrm{Al}$ igual que ocurre con la inteligencia, un factor que se encuentra relacionado con el desarrollo cognitivo es la capacidad de reconocer y actuar de acuerdo con principios morales; en relación con este tema Bennett (1993) señala que el comportamiento moral de una persona está basado en el desarrollo de los fundamentos que lo llevan a la autorrealización plena. Por su parte, esta realización propia se fundamenta tanto en el manejo y estimulación de factores tales como el razonamiento, el carácter, la sensibilidad y la sabiduría, entre otros, como también en las experiencias y educación recibidas, desarrolladas y maduradas a través del tiempo, lo que logra en la persona el carácter y la capacidad de tomar decisiones con autonomía moral.

Ahora bien, Lickona (1992) considera el comportamiento moral como las manifestaciones de honestidad, responsabilidad y respeto desde el individuo como respuesta a situaciones vividas. Entonces, a partir de su experiencia en diferentes ambientes, el individuo va desarrollando identidad social, reflejada posteriormente en actuaciones dentro de la organización, con una visión moral compartida, la cual produce crecimiento individual y organizacional continuo.

Esta perspectiva moral requiere de la educación del carácter que mantendrá las acciones emprendidas en coherencia con los principios personales del individuo, su desarrollo afectivo, el respeto, el desarrollo moral integral entre otros dentro de un contexto global integrativo. En este mismo sentido, para Walker (1999) (citado en Cortés, Pascual, 2002) “... la educación del carácter está adquiriendo una perspectiva integradora promoviendo los valores éticos fundamentales para la formación de la buena persona...". En concordancia con esto, se mencionan algunos aspectos relacionados que serán los que permitan lograr el hábito de la ética personal, a saber: la altitud o valoración propia desde la niñez, la percepción de la identidad personal, la individualidad, la valoración y la independencia; en suma, respecto al desarrollo moral integral es posible decir que el desarrollo de los valores y principios es un factor de gran importancia para el desarrollo de la capacidad cognitiva del individuo.

Cortés Pascual (2002, cap.1) con respecto al futuro comportamental, comenta que los cambios tecnológicos, de información, de crisis sociales, sistémicos externos al individuo e incluso a la sociedad, hacen que el individuo cambie en su desarrollo cognitivo y moral; forzándolo a investigar e innovar y renovar el conocimiento, buscando un equilibrio del desarrollo integral.

Otra manifestación clara del desarrollo cognitivo del individuo es el compromiso, este antecede la acción, es desarrollado y aprendido inicialmente en el hogar, nace desde el interior de la persona, se irradia hacia las personas con las que tiene interrelación; presentándose una situación compleja para el individuo, en donde pone a disposición: capacidades, condiciones y obligaciones en forma coherente e integral, con el fin de cumplir con sus objetivos, dentro de una buena convivencia, dentro de relaciones estables, con responsabilidad y madurez propiciando un efecto directo en la confianza.

Se trae a este contexto la teoría psicosocial de Erik Erikson (1991) referida a la primera infancia, en la que para el niño es muy importante sentir que hay un adulto responsable de cubrir sus necesidades básicas. Desarrollando la confianza, la cual está soportada por la certeza y claridad que se tenga de las capacidades, valores, estructura cognitiva y objetivos en el individuo; contribuyendo para que él asuma posiciones de liderazgo, presente y comunique 
ideas en forma sencilla y competitiva, aportando a su vez al desarrollo cognitivo del mismo.

La confianza hace sentir al individuo seguro, firme, además de incrementar su autoestima (combinando la autonomía, emociones, sentimientos y experiencia de libertad), expresar sus puntos de vista (intelectuales, morales, espirituales), asumir retos y derechos ante la sociedad (se compromete y desarrolla sentido de pertenencia), tomar decisiones e innovar en el entorno en que él se encuentre, estableciendo una interrelación entre la conciencia del ser, sus pensamientos y sus experiencias, alcanzando con éxito sus metas, con hábitos sanos y altruistas; generando productividad en cualquier escenario dentro de un ambiente relacional de confianza interpersonal.

Así las cosas, la organización respecto del individuo debe estar comprometida, ser coherente en sus principios y valores, correspondiendo en forma adecuada y acertada a los esfuerzos realizados por los individuos, a su confianza, con el fin de lograr su desempeño eficiente y eficaz, su liderazgo dentro del contexto social comportamental cultural, alcanzando una imagen diferencial y competitiva respecto de otras organizaciones.

El individuo a su vez en la organización, participa dentro de un entorno social, intercambia experiencias, genera percepciones individuales de confianza propias y hacia sus compañeros, dentro de un ambiente de trabajo profesional, administrando procesos y procedimientos cambiantes, de acuerdo con sus criterios, experiencias y cambios comportamentales organizacionales; manejando una sinergia dentro de los grupos de trabajo, con resultados positivos, promoviendo una cultura de mejora continua, mencionado por Covey (1989).

El intercambio social en una organización, entonces, supone un compromiso de parte de los individuos, dentro de un ambiente de confianza, de respeto, de conocimiento y de aprendizaje mutuos, dentro de directrices justas y equitativas que traerán satisfacciones personales y organizacionales con resultados positivos, en todo caso dejando relaciones sociales efectivas en donde ambas partes ganan.

\subsubsection{El Liderazgo}

El liderazgo es una capacidad especial de los seres humanos, que se enriquece con el proceso de educación recibido y desarrollado por el individuo, el mismo implica tener un ser humano con características diferenciadoras que le permitan legitimar por parte de sus seguidores cualquier toma de decisiones que impliquen acción de grupo.

Partiendo del amor recibido de su hogar, el individuo percibe y aprende a crecer como ser humano, aprovechando sus potencialidades, autoconocimiento, autodisciplina, educado en la necesidad de ir adelante hacia sus metas y poder ejercer su verdadera posición, logrando entender la vida desde diferentes realidades y miradas, llevando a los individuos a ser líderes desde jóvenes, siendo proactivos, audaces y emprendedores; construyendo grupos con nuevas metas y capaces de tomar decisiones en situaciones tanto sencillas como complejas, con impacto social, con paso firme y en beneficio de todos.

Según Richard, Dalf (2006, p. 5) liderazgo es la: “... Relación de influencia que ocurre entre los lideres y sus seguidores, mediante la cual las dos partes pretenden llegar a cambios y resultados reales que reflejen los propósitos que comparten...”. Los líderes, al cooperar con las personas que están a su alrededor, logran producir cambios que generan modificaciones en todas las culturas, incluidas las organizacionales, influyendo en los demás, educando y diseñando nuevos modelos y costumbres. 
Conaty (2009, p. 293) citado por Hellriegel y Slocum, (cap. 10) menciona cinco puntos para tener en cuenta en las organizaciones: evaluar permanentemente al personal, para vitalizar la organización; capacitar continuamente al personal, para evitar el fracaso gerencial; fomentar planes de sucesión de cargos, preparando a otros para dichas funciones; manejar adversidades en forma consistente, directa y enfocada a los objetivos organizacionales y, por último, mantener la imagen de la organización con liderazgo ético y legal.

Por tanto, un buen liderazgo contempla buena comunicación; de tal manera, que se expresen las ideas de forma concreta y directa, produciendo los cambios necesarios, sin traumatismos, elevando estándares morales e intelectuales de los individuos y la organización, con ética, dando como resultado una conducción empresarial mejor, la cual se proyecta en productividad.

Conaty (2009, p. 294), citado por Hellriegel y Slocum (cap. 10), indica “... Los líderes triunfan en la empresa (General Electric) siendo consistentes y directos acerca de varios mensajes fundamentales. Y los mejores no se descarrilan cuando los tiempos se vuelven difíciles...".

Efectivamente, a medida que las organizaciones evolucionan, se requieren líderes con personalidad capaces de superar diferentes riesgos y desafíos, que propongan soluciones innovadoras a los problemas en el desarrollo de las funciones organizacionales. Surgiendo nuevos modelos de liderazgo, a saber:

Liderazgo Transaccional. Hellriegel y Slocum (2009) señalan que su fuerza proviene de la aplicación de procesos administrativos de control, organización y planeación de las metas organizacionales y de las expectativas del individuo en su desempeño a corto plazo. Los líderes transaccionales ofrecen ayudas y engrandecimientos a sus compañeros a cambio de desempeños exitosos; están muy cerca al trabajo desarrollado, son proactivos, evitan errores, son pacientes con los errores, pero son radicales ante desempeños inaceptables; el éxito del liderazgo transaccional se optimiza ante objetivos claros y bien definidos.

En comparación con el transaccional, el liderazgo auténtico, según Hellriegel y Slocum (2009), se caracterizan porque buenos comunicadores se guían por sus valores y creencias, son personas que saben cómo y quiénes son, saben comprenderse a sí mismas, dejan que todos colaboren e incentivan las comunicaciones abiertas y el respeto por las opiniones, decisiones y habilidades individuales, irradiando confianza y credibilidad. Dentro de la organización, establecen diferencias entre metas y competencias, para incentivar habilidades ocultas de su equipo de trabajo, potenciándolo hacia metas comunes, con ética y niveles altos de integridad y honestidad.

Este líder influye en las personas pero respeta la individualidad, valora y estimula el trabajo de los individuos y hace que se sientan satisfechos del deber cumplido. Los resultados en la organización se observan plenamente a largo plazo a través del mejor desempeño.

Como otra perspectiva del liderazgo emergente se considera el liderazgo transformacional. Hellriegel, Slocum, (2009, pp. 301-304) manifiestan que se presenta, en todos los niveles de la organización. Sus miembros se distinguen por ser visionarios, inspiradores, osados y éticos; poseen mente abierta, crean oportunidades, enfrentan y tratan de superar los retos, interactúan positivamente solos o en equipo, aceptan sus habilidades y su creatividad como las de las personas de su alrededor, llegando a satisfacer las necesidades propias y del grupo.

Igualmente, el líder transformacional fomenta la comunicación abierta y clara, la capacidad de escucha, la 
confianza, asume riesgos, fomenta la sinergia del grupo e impulsa la innovación, el grupo hace un esfuerzo y produce una solución en equipo (desarrollo cognitivo) a un problema que individualmente no se resuelve, para llegar a la satisfacción de necesidades de las personas, influenciándolas y persuadiéndolas, produciendo un cambio de cultura.

Otra de las teorías sobre el liderazgo es la del "Liderazgo por Valores", de Luis Gabriel Forero (2010), en la que se establece la importancia de este modelo que deben desarrollar y aplicar los gerentes y empleados, para lograr la excelencia profesional y personal; teniendo en cuenta las competencias y habilidades de las personas exitosas, quienes ejercen un liderazgo fundamentado en valores universales, logrando que los líderes mediante sus valores y su influencia, trasciendan sobre su familia, su empresa y la sociedad en general.

La última de las teorías emergentes de liderazgo es el Modelo Global y la efectividad del comportamiento organizacional, interrelaciona al ser humano integralmente, ocupando un lugar importante en la cultura social, la cultura organizacional y el liderazgo organizacional. El modelo globe (Liderazgo y Cultura México) maneja nueve dimensiones culturales: la asertividad, el colectivismo de grupo, el colectivismo en la sociedad, la orientación al futuro, la equidad de género, la orientación humana, la orientación al desempeño, la distancia al poder y la evasión de la incertidumbre, señalada por Hellriegel y Slocum (2009, cap. 10).

Igualmente, señalan que: “...los valores de la dimensión cultural, no las prácticas, están relacionados con las dimensiones del liderazgo...", enlazan los valores directamente con la cultura y cuando se generan cambios hay desarrollos en cadena, afectando estos cambios al líder, aceptándolas y transmitiéndolas a sus compañeros, aquí se produce automáticamente un impacto hacia la organización, que a la vez realiza un cambio e inicia un nuevo ciclo.

El liderazgo Globe es carismático, autoprotector, autónomo, participativo, va orientado a cada equipo y al ser humano, es decir, parte desde el ser humano y regresa a él. Hellriegel y Slocum (2009, cap. 10) dicen: “...El liderazgo global efectivo implica encontrar soluciones en una forma que permita que los miembros de diversas culturas implementen las decisiones. Aun cuando esto pudiera parecer sencillo, puede ser bastante complicado...”. El líder global es capaz de ser transcultural.

Hellriegel y Slocum (2009, cap. 10) “... Cuando los individuos piensan en las conductas de un líder efectivo, influye más en ellos el valor ('debería ser') que le asignan al futuro deseado que su percepción de las realidades ('como es'), son entonces los lideres un instrumento de cambio social...".

Una vez contempladas las características más importantes del líder en perspectiva al mundo futuro, se hace necesario mencionar la importancia de la creatividad, el reconocimiento, los cambios constructivos y la innovación como excelente legado dejado como consecuencia de liderazgo a una organización o sociedad.

\subsubsection{La creatividad}

Todos los individuos tienen la capacidad para desarrollarla, siendo importantes los incentivos y el entorno en el que viven.

Expresada la creatividad desde muy pequeño, mediante mímica, dibujos, escritos, representaciones, dentro de un entorno al que contribuyen los padres, los profesores, estimulando al niño estableciendo un escenario 
propicio para la creatividad. Lo anterior es mencionado por Martín (1998) cuando habla de creatividad y reforma educativa. De tal manera que unos pueden presentar y representar su creación más fácilmente que otros.

Los padres al romper la monotonía en los juegos hacen que los niños desde su entorno familiar, empiecen a entender cómo funcionan todas las cosas, comenzando a hacer reflexiones, sacar conclusiones y a tratar de cambiar los procedimientos para ver qué resulta, se empieza a desarrollar el espíritu investigador y de creación; dentro de un ambiente de preguntar y aprender; realizando continuas adaptaciones, a través de desequilibrios y equilibrios que de acuerdo con sus valores, aplicación de habilidades y conocimiento, lo llevan a vivir un proceso dinámico de creatividad.

Graham Wallas (1926) presenta modelos de proceso creativo en su trabajo "El arte del pensamiento", relacionados con la preparación (enfoque mental), incubación (interiorización cerebral), intimidación (presentir la solución), iluminación (se da la idea creativa conscientemente), verificación (se aplica la creatividad). Todo lo anterior dice él, le permite al hombre adaptarse a situaciones y evolucionar.

El individuo desarrolla etapas, casi sin percibir conscientemente que lo está haciendo; el enfoque mental lo hará de acuerdo con sus creencias, mirada de la situación y conocimientos, compenetrándose con el problema, construyendo soluciones e incorporando creación; pasando por experiencias que traen nuevos conocimientos, nuevas ideas y crecimiento personal.

Mihaly Csikszentmihalyi (1998) indica: "La creatividad resulta de la interacción de un sistema integrado por tres elementos: una cultura que contiene reglas simbólicas, una persona que traiga la novedad en el dominio simbólico, y un campo de los expertos que reconocen y validan la innovación".

Los individuos para ser creativos conjugan las funciones de su cerebro con las capacidades intelectuales y cognitivas, que hacen que el individuo capte un problema y plantee soluciones adecuadas e innovadoras; analizadas por él dentro de un contexto cultural, aplicando la lógica, la simbología y la razón, aprendiendo de sus experiencias y produciendo el cambio; actuando en un escenario de confianza y aportando nuevas ideas.

Howard Gardner (1999) nos dice que los tres elementos principales de la creatividad son: el individuo, el trabajo y las otras personas; los cuales juegan un papel importante, pues el individuo experimenta situaciones, ordena las ideas en su mente, activa sus pensamientos, asocia las mismas a sus experiencias cognitivas, afectivas, de personalidad, de trabajo y sociales y toma entonces decisiones que producen resultados y cambios, que lo involucran a él a la organización y a los demás de su entorno, de acuerdo con la situación y el momento.

El individuo, entonces, con sus características desarrolla en su mente formas diferentes para convivir adecuadamente, manejando y adaptándose a las situaciones, aprendiendo de las mismas, generando actitudes diferentes, visualizando perspectivas, redefiniendo y tratando situaciones complejas, para evolucionar creativamente.

Alfred Edward Taylor (1901) habla de niveles de creatividad, el expresivo (enfocado hacia el individuo y hacia y desde el entorno); el productivo (referido a la ejecución del mismo); el inventivo (referido a descubrir nuevas situaciones); el innovador (referido a modificación de situaciones) y el emergente (referido a nuevas creaciones). 
Hay que anotar que los problemas cada vez se presentan en escalas y gamas diferentes lo que incorpora una complejidad sistémica, que obliga al individuo a ser más creativo dentro de un mundo globalizado, con incentivos en su motivación que lo llevan a ser exitoso. La capacidad de creación del individuo y los de su entorno crece cuando se descubren nuevas situaciones y a través de la comunicación se generan nuevas técnicas y tácticas, tomando decisiones ajustadas al individuo y al colectivo, creatividad que influye en el grupo social y viceversa.

Como elementos fundamentales se han mencionado en esta parte los factores motivadores que generan procesos creativos, pero que a su vez implican una serie de expectativas para cada individuo, de tal forma que todas sus acciones y todas sus decisiones de orden creativo están dirigidas y estimuladas por factores intrínsecos y experienciales del individuo, razones por las cuales se recuerdan a continuación algunos elementos importantes de la motivación.

\subsubsection{La motivación}

El hombre al nacer viene con un conjunto orgánico fisiológico y social de necesidades, las cuales se van modificando de acuerdo con las costumbres, leyes, normas e ideologías del ámbito al que pertenece; creándose nuevas necesidades que hacen que el individuo cambie sus conductas y comportamientos de acuerdo con una cultura y exprese a su vez de diferentes maneras la parte interna del ser y en especial las que conforman su personalidad.

De la interrelación del individuo con otros individuos, surgen los impulsos conscientes e inconscientes que lo llevan a actuar para satisfacer sus propias necesidades.

En el modelo de Maslow (1963) citado en el libro Motivación y Personalidad se menciona que en todas las situaciones el individuo puede observar que hay varias alternativas, él decidirá por alguna de acuerdo con su personalidad, decisión que lo lleva a ejecutar determinada acción, como un impulso que proviene de su ser, de conformidad con sus experiencias y recuerdos, produciéndose una búsqueda continua de mejores resultados respecto de los objetivos personales, satisfaciendo sus necesidades primarias (referidas al ser) y luego las secundarias (referidas a su parte social y organizacional) dentro de las comunidades en las que vive y que le proporcionan el reconocimiento social.

Desde la niñez se aprende lo que es motivación, a partir de las aprobaciones sociales, observación, imitación, entendimiento y actuar, sintiendo reforzado su aprendizaje con las demostraciones afectivas de sus padres, quienes proporcionan al niño el sentir de las primeras semillas de satisfacción, para que este desarrolle las destrezas necesarias para adelantar el aprender y crecer; para conducirse al cumplimiento sus objetivos y la satisfacción de sus propias necesidades.

Para Gary Dessler (1979): “...La motivación refleja el deseo de una persona de llenar ciertas necesidades..."

Por tanto, el individuo tiene un crecimiento personal dentro de realidades sociales, proporcionado por el continuo aprender, pues los estímulos le despiertan su motivación, aquí se produce una interacción persona-situación. Se originarán en el individuo impulsos, que lo llevan a desarrollar y efectuar acciones eficaces para conseguir sus objetivos (progreso personal), que combinadas con las acciones del colectivo en el caso de una organización, lo conducen a buscar continuamente las mejores alternativas para conseguir las metas proyectadas; dentro de ambientes de cambio y adaptación continuos. 
Luis Gabriel Forero (2010) afirma: “...Motivar es buscar motivos para realizar una tarea, una función o un proyecto, de tal manera que estos motivos lo muevan a realizar su trabajo sin que se sienta obligado sino comprometido...".

El ser humano, a través de la satisfacción de las necesidades establece ciclos motivacionales de carácter personal y social, surgiendo un estado de equilibrio, de compromiso y de madurez respecto a su entorno cultural. Este proceso de maduración tiende a perpetuarse, surgiendo la motivación ante nuevas experiencias, dificultades o procesos de adaptación. De acuerdo con lo anterior, la organización debe proyectar estrategias proactivas para cuando se generen nuevos ciclos adaptativos, ya que estos van a determinar un crecimiento profesional, personal y social que la benefician.

Fredderick Herzberg (2004) citado por Robbins, Stephen, propone que los factores más importantes para tener en cuenta para una mejor administración son, los que tienen que ver con lo que él llama de jerarquía superior, los logros personales y su clase de reconocimiento, la labor específica por cumplir, la responsabilidad de los individuos y sus posibilidades de crecimiento que son sustanciales en una mejor realización organizacional.

Los reconocimientos, las recompensas y el bienestar deben ser estructurados muy deliberadamente por parte de la organización, en un clima de confianza, seguridad y sobretodo de cierto grado de felicidad grupal; de tal manera que los incentivos a los individuos dentro de la organización permitan el desarrollo de las capacidades individuales y grupales.

La empresa efectivamente debe tener en cuenta la cultura en el contexto organizacional, proporcionando al individuo retos que lo podrán potenciar como ser humano y lo podrán conducir a buscar caminos innovadores, convirtiendo la organización en nuevos escenarios de carácter social y profesional dentro de un mundo globalizado, generando nuevas condiciones competitivas. Para conseguir el logro de los objetivos institucionales con un alto nivel de satisfacción, tanto para los individuos como para los equipos de trabajo, creando ambientes susceptibles de mejoramiento operativo y personal. Gordon (2008, citada por Buelvas) menciona: "Las teorías y las investigaciones en el campo de la motivación proporcionan un medio sistemático de diagnosticar el grado de motivación y de recomendar maneras de mejorarla".

Se está, entonces, inmerso en un mundo que evoluciona permanentemente y por ende es necesario renovar y cambiar el enfoque de los incentivos organizacionales, cada vez que la situación lo requiera.

\section{Aspectos referidos a la organización}

A continuación se revisan áreas que al ser combinadas permanentemente dentro de la organización pueden generar un desempeño laboral de crecimiento, plenitud del individuo y desarrollo fortalecido de los equipos de trabajo y la organización.

\subsection{Calidad de vida}

La calidad de vida de un individuo tiene que ver con aquello que es del ser, referido a sentimientos, actitudes, valores, actuaciones, ética, moral, conocimientos y aspiraciones, todos ellos conjugados con las improntas recibidas del entorno, desde su infancia, en el que ha desarrollado sus hábitos, tal como el entorno familiar en donde existen relaciones de compromiso y afecto, las que están presentes y se desarrollan en la medida en que el 
individuo entra en relación con otros entornos de carácter laboral, institucional y afectivo.

$\mathrm{Al}$ producirse lo anterior, como consecuencia en el desempeño laboral hará del individuo una persona más segura, más tranquila, menos aprehensiva, lo cual le permitirá integrarse en equipos, en grupos y en actividades que le generarán algún grado de satisfacción. Es por ello que se hace importante toda reflexión acerca de la incorporación de un individuo estable, satisfecho y operativamente adaptable a cualquier requerimiento social de trabajo. Independientemente de todas las técnicas de selección, un historial de vida estable desde el punto de vista antropológico (referente histórico, referente ético, referente moral, entre otros) hace de él un componente vital a la calidad de la vida organizacional.

Sevila Michel (2011) plantea: “...La calidad de vida en el trabajo es una filosofia, un set de creencias que engloban todos los esfuerzos para incrementar la productividad y mejorar la moral (motivación) de las personas, enfatizando la participación de la gente, la preservación de su dignidad...".

Por lo tanto, la organización debe tener en cuenta que para un desarrollo armónico del individuo se deben acercar profundamente la parte cognitiva del individuo con su vida práctica, igual que la parte moral (colectiva) con la ética (individual), incrementando la gama de posibilidades de acuerdo con la apertura del pensamiento, los entornos y los cambios, conjugados con los factores de la complejidad organizacional, tales como la incertidumbre, la dualidad, la ambigüedad, la subjetividad y las contradicciones, principales puntos de quiebre en las relaciones de fuerzas, los conflictos disfuncionales y las divergencias y las competencias personales y profesionales; a fin de permitir un trabajo más adecuado, digno y con retos personales para cada individuo, proporcionándole una mejor calidad de vida, bajo la teoría del doctor Etkin Jorge (2000, pp. 193 a 214).

Las organizaciones deben desarrollar procesos que estén estrechamente relacionados con los objetivos de la organización y de los individuos, con el fin de lograr que los individuos se acoplen fácilmente a sus normas, necesidades, metas y objetivos. En donde se complementen la responsabilidad, compromiso, competencia individual y esfuerzos para satisfacer necesidades expectativas y aspiraciones del individuo con las de la organización y viceversa; creando situaciones para el individuo atraído por las expectativas del éxito, recompensas por su actuar y toma de decisiones acorde con las conductas hacia el objetivo empresarial.

La calidad de vida organizacional hace aportes a la personalidad y dignidad del individuo, a sus creencias y a los cambios culturales, proporcionando oportunidades de crecimiento y expectativas de mejora. $\mathrm{Al}$ compartir una búsqueda por un mejor futuro entre los individuos de la organización, se genera un mejor ambiente de trabajo, dentro de un sistema dinámico y a la vez flexible que incrementa cada día la productividad de la organización.

La calidad de vida organizacional definitivamente va de la mano con las satisfacciones de los individuos que pertenecen a la organización, con características como buenos salarios, condiciones de trabajo, buen clima organizacional, igualdad de oportunidades, compromiso de la organización como soporte hacia el individuo y expectativas de vida.

\subsection{Entorno laboral}

La fiabilidad de la organización está dada, entre otros, por la confianza entre los individuos que pertenecen a la 
misma y determinan de una forma $\mathrm{u}$ otra las acciones y decisiones. Una de las esencias del entorno laboral proviene de la parte histórica de la organización, pues aquí se evidencian los resultados de las diferentes decisiones, de la influencia de los diferentes propietarios y ejecutivos; y la mezcla de las múltiples culturas y entornos laborales de los que a ella pertenecen y pertenecieron.

En este ambiente laboral resultante los individuos aprenden, se desarrollan y ponen en práctica sus capacidades, dentro de un mundo complejo, adquiriendo compromisos, asumiendo responsabilidades y creando sinergias entre grupos de trabajo.

El individuo se constituye en el eje más importante para una organización, por lo tanto, desde donde se irradia cualquier acción del mismo, él se adapta, se motiva y logra superarse, además de resolver problemas de la organización, pasa la mayor parte de su vida en ese ambiente; comparte personalidad, alegrías, conocimientos, retos, creencias, intercambia sus ideas con las alternativas propuestas por el entorno, intercambia sus relaciones sociales complementándose en forma ideal con las actividades que la organización proporciona, impulsando los resultados organizacionales e individuales.

La organización debe contemplar las mejoras en el entorno laboral de los individuos, rediseñando los puestos de trabajo, incentivándolos con sus programas de capacitación, con expectativas futuras, bienestar físico, emocional y social, contribuyendo a la satisfacción de las necesidades del individuo, de tal manera que este se sienta con compromiso, respeto, confianza, ética; compartiendo trabajos, satisfaciendo necesidades y cumpliendo las metas del individuo y de la organización, trayendo como consecuencia mayor productividad.
Las empresas de hoy tienen cada vez más la necesidad de desarrollar equipos de alto desempeño, para avanzar en sus logros tanto internos como externos a la organización. Uno de los factores más importantes para dinamizar los equipos es la comunicación efectiva, tanto personal como institucional a través del dialogo, por medio del cual se puede detectar los obstáculos e implementar las acciones necesarias para mantener el equilibrio funcional y potenciar la inteligencia de grupo.

\subsection{Entorno intelectual}

Uno de los valores más importantes que poseen las organizaciones es el que resulta de la combinación de las inteligencias de los individuos que pertenecen a la misma; es por eso que las empresas dentro de este mundo globalizado desarrollan mecanismos que les permiten combinar en forma excelente y productiva dichas capacidades.

El entorno intelectual del individuo en la organización está dado no solo por la suma de sus conocimientos y experiencias, sino agregando las prácticas, experticias e investigaciones de los demás miembros de la organización, que combinados con la inteligencia histórica, la información del ambiente externo, los proyectos, estrategias, planes, metas y objetivos por conseguir le permiten desarrollar su propia gestión dentro de un ámbito de crecimiento individual y de la propia organización, crecimiento demarcado por perspectivas, tácticas y operaciones que facilitan actividades creadoras dentro de una contextualización cultural y de toma de decisiones, incrementando el conocimiento y facilitando la innovación, impactando en forma positiva en la inteligencia del individuo y la organización en todos sus niveles.

Hay otras situaciones que tangiblemente se encuentran en el entorno intelectual de los individuos y la organización 
como identidad corporativa, patentes, procesos y procedimientos, tecnología de punta y conocimiento explícito y experiencial de los individuos de la organización. De tal manera, que la organización debe estar atenta, con el fin de capacitar a los individuos para incrementar su nivel de inteligencia, renovación y productividad empresarial; que los llevarán a ser más competitivos y exitosos, redundando en un crecimiento firme de la organización.

Freeman, Stoner y Gilbert (1996) definen a la tecnología y especialmente a la parte de sistemas como: “...mecanismos de control importantes para garantizar las operaciones eficientes en el interior de una organización...".

El desarrollo intelectual permanente que debe realizar el individuo, le permitirá mantener viva la llama de la curiosidad, del entusiasmo por el descubrimiento, además de afianzar su integración y pertenencia, a un círculo intelectual enriquecido con circunstancias de adaptación, asertividad, prospectiva, evolución y cambios para beneficio propio y grupal dentro de su campo laboral.

\subsection{Entorno social}

El entorno social del individuo se compone de una serie de condiciones de vida, emociones, estudios, situación económica, estatus, experiencias que él mismo ha vivido a través del tiempo, de acuerdo con los círculos sociales, por donde ha intercambiado opiniones, necesidades, afecto y sensaciones; entonces, el entorno social estará compuesto por la mezcla de actitudes, expectativas, formas de ser, niveles de inteligencia, costumbres, valores, metas, creencias aprendidas y expresadas, que varían de acuerdo con los diferentes grupos sociales con los que ha interactuado el individuo.
$\mathrm{El}$ individuo cuando pertenece a una organización desarrolla su parte productiva, lo que lo obliga a adaptarse a una serie de modificaciones del entorno social, que implican alteraciones en sus condiciones de vida y exigirá del individuo interacción con otros que difieren de sus intereses, compartiendo sistemas organizacionales de gestión, valores y principios de acción, que modifican o cambian su perspectiva profesional, retos y proyección futura dentro de la misma organización, que lo impulsaran a experiencias nuevas dentro de la organización.

Lev Vygotsky (1997, citado en Baquero) de acuerdo con sus reflexiones afirma: “... el aprendizaje es una forma de apropiación de la herencia cultural disponible, no solo es un proceso individual de asimilación. La interacción social es el origen y el motor del aprendizaje...". Por tanto, la educación compartida contribuye a las interacciones e interrelaciones sociales del individuo, combinadas con otros dentro de un escenario real de convivencia, desarrollando conocimiento mediante enseñanza recíproca, aprendiendo y colaborando mutuamente entre los diferentes entornos sociales organizacionales.

De la interacción social también surgen nuevas costumbres y necesidades por satisfacer, a nivel no solo individual sino grupal y organizacional, razón por la cual se hace necesario que la empresa esté atenta cuando hace la selección de un nuevo empleado, de tal forma que él acepte los nuevos escenarios sociales y fácilmente se adapte a los cambios a través de una interrelación sistemática, promovida por un diseño direccionado de estrategias institucionales, las mismas deben permitir conseguir los objetivos individuales, satisfacer las necesidades grupales y alcanzar los objetivos empresariales.

La organización, por tanto, debe tener un esquema predeterminado de valores y principios que soporten las conductas, contar con un plan estratégico definido para 
implementar los elementos organizacionales que hacen que el individuo y la organización se desarrollen en términos de su expresión personal y profesional, a través de la integración de equipos de alto desempeño enfocados en sus propios logros y en los de la organización. Esta nueva cultura organizacional del desarrollo compartido generará en el individuo compromisos, motivos y valores que dan identidad individual y de grupo, que lo proyectarán en una sociedad más digna, capaces de tomar decisiones trascendentales con proyección social y e institucional.

La tarea de la organización por ser tan compleja necesita de comprensión holística, entre lo que se puede llamar fenómeno humano y continuos cambios del individuo de acuerdo con los entornos, en este caso la organización; cambios culturales que pueden desdibujarse por las formas de interacción del individuo, por estar inmersa la tecnología, ciencia, los cambios intelectuales entre compañeros y los cambios que se construyen socialmente en la organización, generando exigencia en competencia de capacidades y asimilación al cambio.

\section{Desarrollo organizacional}

Las organizaciones se desempeñan en entornos cambiantes que las obligan a transformase continuamente y además, estos cambios les exigen desarrollar herramientas y mecanismos para poder adaptarse óptimamente en momentos y tiempos difíciles y retadores.

Beckhard (1972) define el desarrollo organizacional como: “... Un esfuerzo planificado de toda la organización y administrado desde la alta gerencia, para aumentar la efectividad y el bienestar de la organización por medio de intervenciones planificadas en los procesos de la entidad, los cuales aplican los conocimientos de las ciencias del comportamiento...".
Muchinsky (1994, citado en Axelrod 2004) manifiesta que el desarrollo organizacional es una subdisciplina que evoluciona muy rápidamente, siendo este un esfuerzo de toda la organización que debe ser apoyado por la alta gerencia.

En el desarrollo organizacional, uno de los objetivos esenciales es el recurso humano, en cuanto a su formación, participación y contribución a los objetivos organizacionales sin olvidar sus propios objetivos, es por eso, que es necesario direccionar desde el nivel ejecutivo, no solo la selección del individuo adecuado a una labor o desempeño, sino que es necesario visualizar un plan de desarrollo individual y grupal partiendo de sus potencialidades y competencias pero enfocadas en el plan estratégico de fortalecimiento y desempeño organizacional, en busca de unos resultados óptimos y un crecimiento permanente de las características y las capacidades potencialmente desarrollables con nuevas enseñanzas y entrenamiento organizacional.

La organización dentro de su desarrollo, debe establecer mecanismos de mejora continua, renovando procesos organizacionales que generan renovación de cultura permanentemente, soportado en el individuo y sus características, con un norte definido desde el punto de vista del individuo y de la organización; mediante procedimientos flexibles, autorregulados, dando reconocimientos a los individuos y ejerciendo un liderazgo transformacional, con una estructura social basada en el respeto y la buena comunicación.

El desarrollo organizacional permite tener una prospectiva de la organización, basada en la integración del individuo al desempeño global de la organización, mediante el compromiso del logro personal enfocado al logro organizacional. Facilitando las actividades y el control de las acciones hacia el objetivo de la organización (planes 
precisos, claros y compatibles con la necesidad de los individuos), para facilitar el cumplimiento de los proyectos; asegurando el éxito misional, logrando que los individuos, equipos de trabajo y organización funcionen constructivamente con el compromiso de todos (Kenneth, 1992).

Hay que entender que para lograr una estabilidad evolutiva organizacional es necesario diseñar y ejecutar planes de mejoramiento continuo, armonizando y balanceando la resistencia del personal y del nivel directivo; proporcionando una respuesta eficiente, flexible y proactiva que renueve permanentemente conocimientos y experiencias de equipos de trabajo, combinando los elementos individuales con la totalidad de la organización, logrando un manejo correcto de los conflictos para tomar decisiones acertadas con visión a políticas y objetivos organizacionales, construyendo a su vez desarrollo organizacional.

\section{Comportamiento organizacional}

El individuo en la organización es el punto de partida, es por eso que desde allí debe observar y entender lo que se denomina comportamiento organizacional, puesto que el individuo tiene su propio desarrollo, necesidades, forma de comportamiento y experiencias, que lo llevan a valorar con madurez la organización, establecer sus compromisos y visualizar su futuro.

El comportamiento organizacional se relaciona con el sentir y actuar del ser humano en el lugar de trabajo, su comportamiento, interrelación e interacción entre las personas y la organización y la organización misma. "...Es una disciplina que investiga la influencia que los individuos, grupos y estructura ejercen sobre la conducta dentro de las organizaciones, a fin de aplicar esos conocimientos para el desarrollo de estas..." (comentado por Gibson, Ivancevich y Donnelly, 1992).

Chiavenato Idalberto (2004), respecto de la conducta del ser humano, agrupa el comportamiento en tres premisas: primera, el comportamiento causado (interno y externo al individuo), segunda, motivación (relacionada con sus necesidades); y por último, el referido al comportamiento hacia los objetivos (referida a que primará la conducta dirigida del individuo), de tal forma que el trabajo y la visión del mismo alimentan la organización y retroalimentan del individuo con el fin de conseguir sus metas.

La organización, entonces, debe estar atenta a los cambios conductuales que transforman el comportamiento de los individuos, puesto que los mismos están correlacionados con los demás de la organización y tienen un efecto directo en los resultados de la misma, por sus impactos, resultados, procesos y gestiones.

El individuo debe ser el centro de la organización, por tanto, alrededor de este se desarrollan una serie de acontecimientos que sumados a las características de la organización dentro de un ambiente social darán una cultura organizacional que aglomera estos comportamientos (Daft y Steers, 1992).

Dentro del comportamiento organizacional también se refleja la influencia de los diferentes dueños, la antigüedad de los individuos que trabajan para ella, las consecuencias de las decisiones y el aporte de los líderes y las personas especiales que han propiciado los cambios de la organización.

Stephen P. Robbins (2004) relaciona el comportamiento organizacional como "... un campo de estudio que investiga el impacto de los individuos, grupos y estructuras sobre el comportamiento dentro de las organizaciones, 
con el propósito de aplicar los conocimientos adquiridos en la mejora de la eficacia de una organización...".

El comportamiento organizacional refleja cuál es el lenguaje comúnmente utilizado por los individuos para comunicarse con sus compañeros dentro y hacia los grupos de trabajo, así como la responsabilidad y el comportamiento de sus miembros frente a una situación, igual que la retroalimentación y acciones de mejoramiento para la toma de decisiones ante nuevas competencias, con altos índices de calidad, estableciendo ajustes en los procedimientos y adaptándose en forma rápida en el interior de la organización, con el objetivo de cumplir las metas establecidas para el fin común organizacional.

El comportamiento organizacional contempla todas las características y habilidades del ser humano, no solo profesionales sino intelectuales, administrativas y sociales, dentro de un ambiente cambiante. La organización con la visión de optimizar su capital humano, debe proyectar la capacitación de su personal de acuerdo con las nuevas necesidades, esta situación lleva inmerso un cambio cultural organizacional, siendo necesario un nivel de respuesta a los cambios del entorno más eficiente, generando resultados con mayor celeridad que aquella organización que no defina estos planes.

Stephen P. Robbins (2003) contempla en el comportamiento organizacional tres niveles de análisis desde el punto de vista del individuo: la persona como tal, personalidad, en cuanto a autoestima, autocontrol, valores, habilidades, motivación, a nivel grupal procesos sociales y dirección de grupo en cuanto a procesos evolutivos, contingencias y toma de decisiones y a nivel organizacional desde el punto de vista de el qué y el para qué, es la organización, cómo funciona la dirección y la comprensión de procesos y problemas organizativos.
En síntesis, toda organización posee un comportamiento organizacional cambiante, que depende del comportamiento del individuo y sus miembros, de las funciones y acciones de cada uno, percibidas y modificadas de acuerdo con los impactos del entorno, generando seguridad, estabilidad y satisfacción, en donde se reflejarán los resultados perseguidos y la visión de cada organización respecto de su capital humano.

\subsection{Cultura organizacional}

La cultura en cualquier sitio recoge el rastro histórico del individuo, es decir, todo lo que tiene que ver con las cualidades, relaciones y capacidades del individuo, que sumadas con su parte espiritual y material, incluidos la educación, los conocimientos, las experiencias, los sentimientos, las creencias, los valores y los principios morales, además de las expresiones, la creatividad, la responsabilidad, la investigación, los resultados de las experiencias con otros individuos en donde necesariamente se establece una dinámica de costumbres, reconocimientos y estímulos tanto positivos como negativos que dan como resultado un comportamiento de carácter social.

Gordon (2006, citado por Gutiérrez) afirma “...La cultura de una organización describe la parte de su ambiente interno que incorpora la serie de supuestos, creencias y valores que comparten los miembros de la organización y que usan para guiar su funcionamiento...".

Complementando lo anterior Chiavenato (1989) muestra la cultura organizacional como "...un modo de vida, un sistema de creencias y valores, una forma aceptada de interacción y relaciones típicas de determinada organización..." (p. 464). 
Se puede afirmar que las creencias y los valores de los individuos se integran dando como resultado un comportamiento que se convierte en cultural, los comportamientos generados por esta cultura, dentro de un escenario organizacional unen a las personas socialmente, de tal forma que esta cultura se encarga de transmitir identidad, compromiso, estabilidad, supuestos sociales, conocimientos, creencias, actitudes y en definitiva el actuar de los individuos que pertenecen a la organización, incluyendo las características propuestas por la organización a través de sus estilos de administración, su toma de decisiones que son definidas por sus políticas, teniendo como resultado estrategias que garantizan la productividad y el éxito.

Asimismo, como dentro de la organización se viven y desarrollan gran cantidad de procesos, caracterizados por símbolos de autonomía, decisiones, capacidades disponibles, resultados de investigaciones, la solución a problemas, nuevos conocimientos, la apropiación y aplicación de nuevas tecnologías, la retroalimentación a procesos y procedimientos, la auto renovación, todos ellos integrados traen como resultado cambios de pensamientos y comportamientos de los individuos que generan una nueva y única cultura organizacional y por tanto una identidad organizacional única.

Tenemos entonces, que la organización evolucionará y como consecuencia se desarrollará un liderazgo transformacional, produciendo mejores resultados, asumiendo positivamente sus aciertos y desaciertos de tal manera que los pueda explotar como fortalezas competitivas, dentro de un cambio permanente y proactivo para la organización.

Desde el punto de vista de la organización, los cambios producidos por la relación entre sus miembros, en los grupos de trabajo, respecto a otros grupos de trabajo y a otras organizaciones, la sumergen dentro de una gran diversidad cultural y ocasionan un crecimiento propio y autónomo de la organización. Respecto a otras organizaciones de su campo de trabajo y por las culturas que de ellas comparten, hacen que se diferencie de otros sectores de desempeño. En todo caso su diferenciación siempre estará determinada por los individuos que la componen, por sus características culturales y experienciales dándole una identidad competitiva en un sistema complejo de mercado.

\section{Conclusiones}

El individuo a través de la vida se va estructurando en su parte física, moral, intelectual, cognitiva y experiencial, creciendo integralmente en la medida que tiene contacto con los diferentes ambientes y ámbitos; sin embargo, todo esto va siempre matizado con sus emociones, percepciones, intuiciones y reacciones que ratifican tener unos resultados únicos e irrepetibles de madurez, de satisfacción personal y de crecimiento integrado al mundo que lo rodea. Estos resultados al ser capitalizados por la organización permitirán un desarrollo y un crecimiento continuo de este ser humano que será intencionalmente conducido al éxito personal y por ende al éxito organizacional.

La educación formal, la educación familiar y el aprendizaje dirigido del individuo determinan una mejora en sus habilidades tanto verbales como no verbales; los rasgos actitudinales, la confianza personal y su compromiso respecto a determinadas situaciones regulan una conducta acorde con los estímulos percibidos; de tal manera, que puede neutralizar incertidumbres y controlar interpretaciones en los diferentes entornos culturales; recibiendo y aportando en forma óptima a las competencias interculturales, perfeccionando su parte cognitiva, interpretando las experiencias de las otras personas, con sus propias experiencias, sentimientos, razones, conocimientos y moral que combinadas con los resultados sociales reflejarán un 
comportamiento ético, encaminado a un equilibrio personal y social que se refleja en un compromiso holístico social.

La combinación de la integralidad del ser humano, con las relaciones interpersonales, la adaptabilidad y la capacidad para responder ante una nueva situación en forma efectiva, en especial en el ámbito organizacional dará como resultado una ventaja competitiva de evolución de la organización respecto de las demás organizaciones, determinando una capacidad y calidad de respuesta llena de decisiones acertadas.

El ser humano integral, su experiencia, el respeto por la individualidad, sus aportes a la sociedad, lo llevan a ser un buen crítico de las realidades, del entorno y en este caso de la organización; llegando a potenciar la comprensión de todas las complejidades del mundo, a adaptarse al cambio evolutivo, en forma individual y como eje activo de la organización, de tal manera que representa como un todo a su grupo de trabajo en forma proactiva, capitalizando las oportunidades a través de la competitividad y dentro de un crecimiento continuo, siendo generador del cambio a través de valores.

Para lograr un perfeccionamiento como seres humanos y como organización se hace necesario optimizar los procesos educativos, mediante mejoras continuas, en autonomía, en calidad de vida del individuo, comunicación corporativa, liderazgo transformacional y el grado de madurez del pensamiento y las capacidades emocionales e intelectuales de quienes la componen. Es así, que el individuo y las organizaciones tienen su propia identidad, compartiendo creencias, capacidades, valores, ambientes cambiantes tanto internos como externos. A su vez establecen una serie de interrelaciones y elaboran nuevos modelos mentales y creativos que los llevarán a innovar y solucionar los problemas propiciando un verdadero cambio social.

Las metas y objetivos organizacionales soportadas en una buena planificación, buen sistema de información, buena comunicación, deben permitir la participación activa del individuo con todas sus competencias laborales, intelectuales, afectivas, de pensamiento, de compromiso y profesionalismo, para llevar la organización al logro de las perspectivas de visión futura global, con una verdadera prospectiva de estrategias por desarrollar, tanto en los campos tecnológicos como del talento humano y su participación positiva en mercados altamente competitivos.

La visión cultural del individuo y la organización dentro de los diferentes entornos sociales es cambiante, influye en los resultados de comportamiento organizacional, que se reflejan en los ciclos productivos del individuo y la organización. Sin embargo, la organización debe intencionalmente generar una cultura, para mantener y mejorar los valores individuales e institucionales, que soporten cualquier tipo de estructura o reto sin resquebrajar la estructura social de la productividad, de la eficiencia, de la evolución y de la innovación y creatividad personal.

Las organizaciones deben diseñar escenarios propicios tendientes al buen manejo y adaptación de los individuos, para que ellos puedan entender y adaptarse a los impactos externos e internos que alteran a la organización, generando a su vez relaciones armónicas, y competitividad en el establecimiento de nuevas estrategias entre grupos de trabajo, para llegar a cumplir las metas organizacionales, regulando experiencias aprendidas y equilibrando las habilidades de los individuos de tal manera que se produzca una dinámica organizacional controlada y una evolución con altos beneficios para todos. 


\section{Referencias}

Axelrod. (2004). Psychologies Labored, 21. Gilbert y Tang, 1998, European Journal of Social Sciences, vol 8, number 2, 2009.

Baquero, R. (1997). Vygotsky y el aprendizaje escolar. (cap. 1. El aprendizaje). Buenos Aires: Editorial Atique.

Beckhard, R. (1972). Desarrollo organizacional, estrategias y modelos. México: Fondo Educativo Interamericano en: http://www.excelencia.uat.edu. $\mathrm{mx} /$ pariente/Articulos/Administracion/Pariente_ DO.pdf. Desarrollo Organizacional,José Luis Pariente F, Capitulo 5 Lecturas

Becker, G. (1975). Human Capital. A. Theoretical and Empirical Analysis, with especial reference to Education. New York: Publicado por acuerdo con Columbia University Press, second edition.

Bennett, J. (1993). Book of virtues, Bellevue, WA: S\&S Trade Borrego de Dios, S (1999), Desarrollo socio-personal y moral la cara interna de la educación global.

Buelvas, J. (Septiembre, 2008). Piensa conmigo guajira. (Ensayo sobre Motivación Laboral), Jimmy E. Buelvas Jurado en: http://jimmyebuelvasjurado.blogspot. com/2008_09_01_archive.html

Chiavenato, I. (1989). Introducción a la Teoría General de la Administración. México: Mc. Graw-Hill Interamericana de México.

Chiavenato, I. (2004). Comportamiento organizacional, la dinámica del éxito en las organizaciones. México: Thomson Learning.
Cortes Pascual, A. (2002). Implicaciones psicopedagógicas de un desarrollo moral integro: La educación holística. Revista Iberoamericana de Educación, en http:// www.rieoei.org/de los lectores/445Cortes.pdf.

Cortes Pascual, A. (2002). Hacia un modelo global de desarrollo moral desde Kohlberg y Bronfenbrenner. (Cap. 1). Bilbao: Servicios de Publicaciones de la UPV.

Covey, S. (1989). Los siete hábitos de la gente altamente efectiva. Publicado inicialmente en 1989, en inglés, (titulado originalmente The Seven Habits of Highly Effective People). Barcelona: Paidós Ibérica.

Csikszentmihalyi, M. (1998). Creatividad. El fluir y la psicología del descubrimiento y la invención. Barcelona: Editorial Paidós.

Daft, R.L. y Steers, R.M. (1992). El Comportamiento del individuo y de los grupos humanos. México: Limusa.

Dalf, R. (2006). Experiencia del Liderazgo. (3ª. ed., cap. 1). México: Editorial Thompson Corporation.

Dessler, G. (2001).Administraciónde Personal. (8 ${ }^{\mathrm{a}}$. ed.). México, D.F.: Pearson Prentice Hall Hispanoamericana.

Erikson, E. (Mayo, 2010). El Desarrollo del niño, las etapas de su desarrollo vital de acuerdo a la teoría psicosocial del desarrollo humano de Erik Erikson, 1991. en: http://www. network-press.org//erikson.

Etkin, J. (2000). Política gobierno y gerencia de la organización. (cap.3). Chile: Pearson Education.

Forero, L.G. (2010). Liderazgo por valores. (3 ${ }^{\mathrm{a}}$ ed.). Bogotá, Colombia: Editorial Buena Semilla. www.diagnosticaescuelaliderazgo.com. 
Freeman, G. (1996). Administración Stoner. (6 ${ }^{\mathrm{a}}$ ed.). México: Editorial Prentice hall Hispanoamérica.

Gardner, H. (1993). Mentes creativas. Barcelona: Paidós Ibérica.

Gardner, H. (1999). Mentes extraordinarias. Barcelona: Editorial Kairós.

Gardner, H. (2001). La inteligencia reformulada, las inteligencias múltiples en el siglo XXI. Barcelona: Ediciones Paidós.

Gibson, J., Ivancevich, J., Donnelly J. Jr. (1992). Las organizaciones: comportamiento, estructura, procesos. $\left(7^{\mathrm{a}}\right.$ ed.). Delaware. U. S. A.: Editorial Addison-Wesley Iberoamericana. Citado trabajo Final de Graduación El caso de la Autoridad Reguladora de los Servicios Públicos Dehisha Broomfield Thompson y Arlene González Castillo, 2007. http:www.uned.ac.cr/sep/ recursos/investigaciones/documents/MicroslfWord

González, R. (2007). Comportamiento organizacional con otras disciplinas. En Revista Consulta Furídica de Chile, La Consulta Preventiva, Friday, June 29, 2007. en: consultajuridicachile.blogspot.com/2007/08/comportamiento-organizacional-y.html

Gutiérrez, E. (2007, enero-abril). Cultura, organizaciones e intervención, 6(1), 122. Bogotá: Pontificia Universidad Javeriana. http:77sparta.javeriana.edu.co/psicologia7publicaciones7actualizarrevista7archivos7v6N110. pdf

Hellriegel, D. \& Slocum, J. Jr. (2009). Comportamiento organizacional, liderazgo efectivo, nuevas perspectivas, aprender de la experiencia. México: Editorial South -Western Cengage Learning (12 ed., cap.10) pp. 292-317.
Kenneth, J. (1992). Manual de administración estratégica. México: Ediciones McGraw-Hill.

Kohlberg, L. (1992). Psicología del desarrollo moral. Bilbao: Editorial Descleè de Brouwer. (Trabajo original publicado en 1984).

Lickona, T. (1992). Educating for character: How our teach respect and responsibility. New York: Bantam Book.

Martín, R. (1998). Creatividad y reforma educativa. Santiago de Compostela: ED Servicio de Publicaciones e Intercambio Científico, Campus Universitario Sur.

Maslow, A. (1963). Motivación y personalidad. Editorial Sagitario.

Meyer, J. P y Allen, N J. (1991). A three component conceptualization of organizational commitment. Human Resource Management Reviere, 1, (pp. 61-98).

Myers, D. (2006). Psicología, (3 ${ }^{\mathrm{a}}$ ed.). Madrid: Editorial Médica Panamericana.

Oliva, A. y Parra, A. (2001). Autonomía emocional durante la adolescencia infancia y aprendizaje 24(2), 181-196. Universidad de Sevilla. http://personal.us.es/oliva/ AE.pdf

Robbins, S. (2003). Comportamiento organizacional. Conceptos, controversias y aplicaciones. México: Pearson-PrenticeHall (10 ed.).

Robbins, S. (2004). Comportamiento organizacional. (10 ed.). México: Editorial Prentice Hall.

Sevilla, R. (4, marzo, 2011,). Desarrollo organizacional y calidad de vida en el trabajo. (Cap. II). Proyectos de 
Importancia del desarrollo de los individuos y su incidencia en el comportamiento organizacional María Clemencia Ardila Franco, Ana Elsa Vargas Espinosa

Calidad de Vida, numeral 2.1 Definiciones. Documento 6948262. http;//200.34.41.59/portafolio/desarrollo. htm pag. 2

Taylor, A. (1901). The Problem of conduct, a study in the phenomenology of ethics. London: MacMillan and Co. Limited, New York: The MacMillan Company.

Vidal, J. (2007). El desarrollo del ser humano. Aceb.org. http://www.aceb.org/desarr.htm

Wallas, G. (1926). The Art of Thought, New York: Harcourt-Brace. 